\title{
Use of novel metallic nanoparticles for improvisation of electrical properties of PVDF-HFP polymer film
}

\author{
A. L. Gayen ${ }^{1}$,.B. K. Paul ${ }^{1,2}$, S. Das ${ }^{1,3}$, D. Mondal ${ }^{3}$, D . Roy ${ }^{1}$, P. Bandyopadhyay ${ }^{1,3}$, S. Manna ${ }^{1}$, \\ R. Basu, ${ }^{1,4}$, D. S. Bhar ${ }^{1}$, P. Nandy ${ }^{1}$ \\ ${ }^{1}$ Centre for Interdisciplinary Research and Education, Kolkata 700 068, India. \\ ${ }^{2}$ Central Glass \& Ceramic Research Institute, Kolkata 700032 \\ ${ }^{3}$ Physics Department, Jadavpur University, Kolkata 700 032, India \\ ${ }^{4}$ Physics Department, Jogamaya Devi College, Kolkata 700 026, India
}

\begin{abstract}
Nanomaterials of compound of copper and arsenic were formed in alcohol in a novel way. Incorporation of these materials by simple solution casting technique in poly vinylidene fluoride-co-hexafluoropropylene (PVDF-HFP) polymer films significantly improves their electrical properties.

The incorporation of the nanomaterial in the polymer matrix activates the transition of phase between $\alpha$ and $\beta$. This provides higher mobility of the charge carriers which participate in the interfacial polarization. These films have higher dielectric constant, ac conductivity and lower dissipation factor $(\tan \delta)$ at room temperature, compared to the pure PVDF-HFP.

These nanocomposites are easy to fabricate and environment friendly and their presence in polymer matrix to get enhanced electrical properties will have a significant contribution in the present day research in electronics.
\end{abstract}

Poly(vinylidene fluoride) (PVDF) and its co-polymers like poly(vinylidene fluoride-hexafluoropropylene) (PVDF-HFP) are in great demand for their versatile and unique properties like flexibility, low processing temperature, low dielectric constant, high dielectric breakdown field etc., making them potential candidate for a broad range of applications in electronic industry.

In order to improve the capacitive performance of the polymer material, a great deal of effort had been devoted to develop PVDF-HFP composites by incorporating different metallic nanoparticles within the matrix. The effective dielectric permittivity of these metal nanoparticles (NPs) doped polymer composite are higher than that of the host polymer matrix. They also show enhancement of conductivity and decrement of tangent loss making them potential candidates as good capacitors and electric energy storage devices. 
The compound of Copper and Arsenic (Cu-As) at different potencies were obtained from Hahnemann Publishing Company, India. The NP aspect of these potencies has been proved experimentally. The advantage of this method is that in this way the shape and size of the NPs can be regulated by the method of preparation. Moreover, these NPs are eco-friendly, inexpensive, nontoxic, easily available metallic nano fillers which can enhance the dielectric constant, conductivity and decreases the dissipation factor of polymer films by many folds.

These NPs were incorporated in the polymer PVDF-HFP (Sigma-Aldrich, USA) in dimethylsulfoxoide solution (Merck, India) and films were prepared by simple solution casting technique. In a typical synthesis, $100 \mathrm{mg}$ of PVDF-HFP was added to $2 \mathrm{ml}$ DMSO and mixed together under vigorous stirring at $60{ }^{\circ} \mathrm{C}$ for $3 \mathrm{~h}$. Measured amount of $\mathrm{Cu}$-As at different potencies were added to the solution. The volume wt $\%$ of all prepared NPs of different potencies is $23.08 \%$. CuAs PC were obtained by casting the whole mixture in clean dry petri dishes and evaporating the solvent in an incubated oven at $60{ }^{\circ} \mathrm{C}$ for $12 \mathrm{~h}$. The films were then coated by silver paste on both sides for electrical measurements. The synthesized films had the thickness in the range of $48-54 \mu \mathrm{m}$ as measured by using a digital screw gauge. The average density value of the fabricated films is $0.81 \mathrm{gm} \mathrm{cm}^{-3}$.

These nanocomposites of PVDF-HFP/Cu-As (Cu-As PC) were studied by EDX (Fig.1). The enhancement of dielectric constant and conductivity and decrease of tangent loss of the nanocomposite films were observed by changing the potency and the observed values were compared with the pure PVDF-HFP film.

At lower frequency the easy orientation of dipoles as well as interfacial polarization contribute enhancement in dielectric constant (Fig. 2). As the frequency is increased further, dipole response is restricted and the dielectric constant has a saturation tendency. In this case, the internal individual dipoles contribute to the dielectric constant which is ideally the electronic polarisation effect (1).

Throughout the frequency range, tangent loss continuously decreases with increasing frequency for all films (Fig. 2). This rapid decrement may be due to intermolecular friction or vibration. At lower frequency range the easy orientation of dipoles for higher relaxation time contributes to higher tangent loss. As frequency increases, less polarization effect continues due to less relaxation time. So intermolecular friction or vibration diminishes which is responsible for saturated tangent loss. On the other hand after $100 \mathrm{KHz}$ the tangent loss increases for all films which may be due to leakage current.

The ac conductivity increases exponentially with frequency for all the nanocomposite films (Fig.2). This increase in conductivity with frequency arises due to the presence of free ions in the polymer matrix that may increase the mobility of the ions which finds an easy path to move and hence increase the electrical conductivity. It has also been observed that the value of ac conductivity is higher for all nanocomposite films compared to the pure polymer film. The increase of $\sigma_{\mathrm{a} . \mathrm{c}}$ is due to the increased aspect ratio of metal ions (1). 


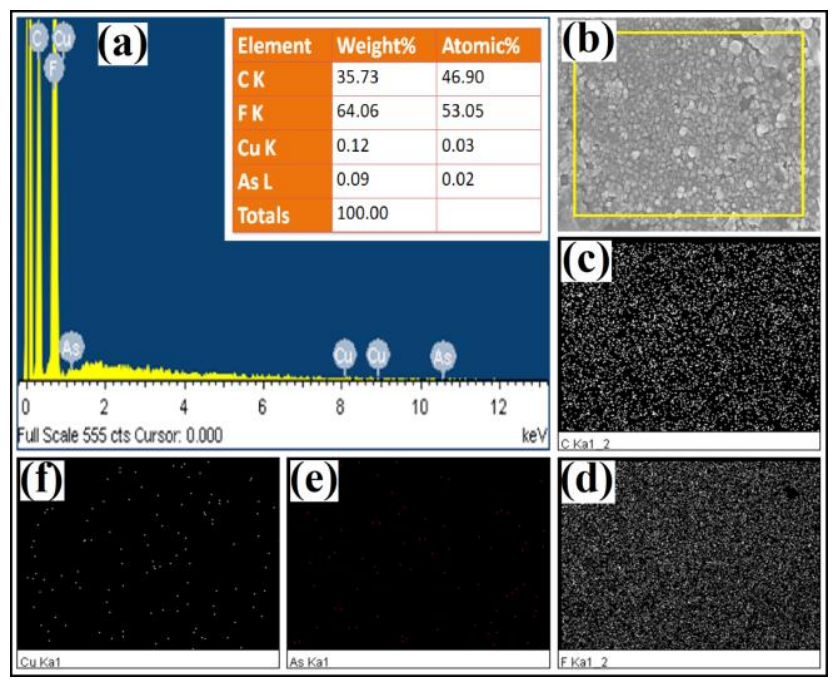

Figure 1. EDX analysis and elemental mapping of $\mathrm{Cu}-\mathrm{As} \mathrm{PC}$

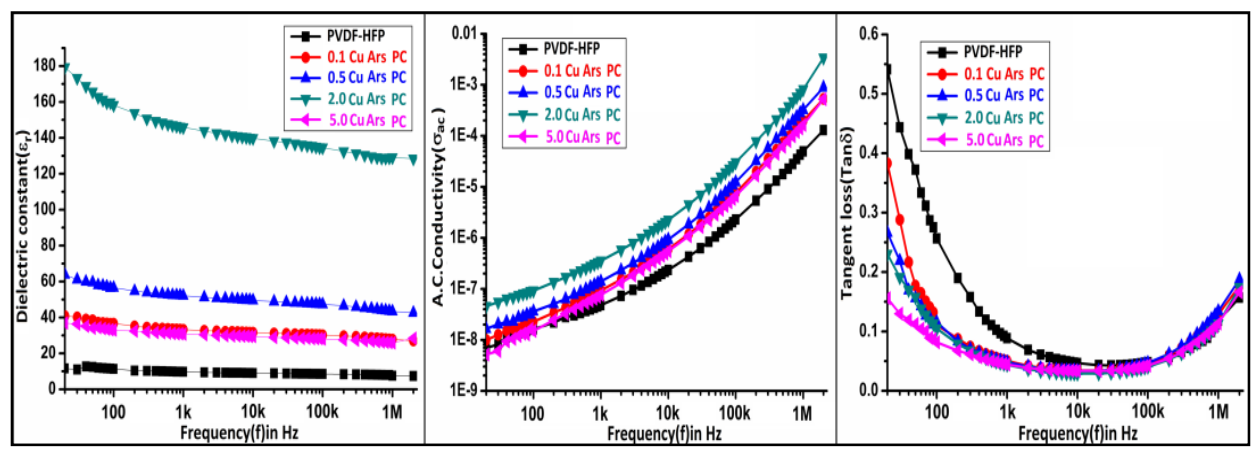

Figure 2. Electrical characteristics of $\mathrm{Cu}$-Ars compound

We conclude that pure polymer films which have comparatively low dielectric constant can be modified into materials with enhanced dielectric constant and comparatively low tangent loss by making a composite with specially devised metal NP and hence can be a promising candidate for the fabrication of high charge- storing multilayer capacitors.

1. A. L. Gayen, D. Mondal, B. K. Paul, D. Roy, P. Bandyopadhyay, S. Manna, R. Basu, S. Das, D. S. Bhar, P. Nandy

Improvisation of electrical properties of PVDF-HFP: use of novel metallic nanoparticles 2017 J. Material Science: Materials in Electronics 28:14798-14808 